\title{
Analyzing Source Sampling to Reduce Error in ECG Forward Simulations
}

\author{
Jess Tate $^{1}$, Karli Gillette ${ }^{2}$, Brett Burton ${ }^{1}$, Wilson Good ${ }^{1}$, Jaume Coll-Font ${ }^{3}$, \\ Dana Brooks ${ }^{3}$, Rob MacLeod ${ }^{1,2}$ \\ ${ }^{1}$ University of Utah, Salt Lake City, Utah, USA \\ ${ }^{2}$ Graz University, Graz, Austria \\ ${ }^{3}$ Northeastern University, Boston, MA, USA
}

\begin{abstract}
A continuing challenge in validating ECG Imaging is the persistent error in the associated forward problem observed in experimental studies. One possible cause of error is insufficient representation of the cardiac sources, which is often measured from only the ventricular epicardium, ignoring the endocardium and the atria. We hypothesize that measurements that completely cover the heart are required for accurate forward solutions. In this study, we used simulated and measured cardiac potentials to test the effect of different levels of sampling on the forward simulation. We found that omitting source samples on the atria increases the peak RMS error by a mean of $464 \mu V$ when compared the the fully sampled cardiac surface. Increasing the sampling on the atria in stages reduced the average error of the forward simulation proportionally to the number of additional samples and revealed some strategies may reduce error with fewer samples, such as adding samples to the $A V$ plane and the atrial roof. Based on these results, we can design a sampling strategy to use in future validation studies.
\end{abstract}

\section{Introduction}

Electrocardiographic Imaging (ECGI) is a promising field for noninvasive diagnosis and treatment of cardiac arrhythmias. ECGI relies heavily on the accuracy of the ECG forward simulation [1], and although this simulation is mathematically relatively simple, existing validation studies have shown greater error than expected [2], which may also be present in ECGI. Therefore, improving validation of the ECG forward simulations will also improve validation of ECGI.

Potential errors in the experimental validation of the ECG forward simulation are introduced from many stages in the process, but one yet under explored origin of error is the cardiac source spatial sampling. Due to technical and practical limitations, researchers performing val- idation experiments must often choose between sampling density and coverage of the cardiac sources. The mathematical relationship between these sources and the body surface potential maps (BSPM) [1] indicates that any missing cardiac sources could have a significant effect on the forward simulation and could cause discrepancies in simulated and measured BSPM.

Many validation studies have used a ventricular epicardial sock of electrodes to record epicardial extracellular potentials. These socks allow for relatively high spatial sampling and coverage of the ventricular epicardium, yet exclude sampling of the atrial epicardium $[2,3]$. Recordings from this region of the heart would include possible atrial or ventricular endocardial sources from the forward simulation; excluding them can introduce substantial error in the forward simulation. Understanding the effect of absent recordings in the atrial region of the heart can help drive strategies to improve validation studies of ECG forward simulations.

In this study, we compared the effect of various atrial region sampling strategies combined with a ventricular sock on the ECG forward simulation. We hypothesized that measurements that from the atrial region of the heart are necessary for accurate forward solutions. Additionally, we want to design a sampling strategy that may be employed in a validation experiment to minimize error resulting from incomplete sampling of cardiac sources. Reducing errors from source sampling will help increase the accuracy of the ECG forward simulation and ultimately the accuracy of ECGI.

\section{Methods}

We analyzed the effect of sampling strategies of the atrial region in ECG forward simulation by sampling the cardiac source in numerous ways, then using those sources in the simulation pipeline. We tested these sampling strategies on three different geometries and source models; two simulated cardiac source datasets (the CARP and KIT) and one experimentally recorded dataset (cage). The ECG for- 
ward simulations from the subsampled sources were compared to fully sampled FEM simulations.

\subsection{Datasets}

The CARP dataset used the pseudo-bidomain method in CARP [4] to simulate the extracellular potentials in an isolated heart with four activation patterns: sinus, RV free wall pacing, LV free wall pacing, and apical pacing. An ellipsoidal cap was placed on a subsampled mesh of the epicardium to generate an enclosed pericardial mesh for the ECG forward simulation.

The KIT dataset heart and torso geometry was generated from a patient scan [5]. Four activation patterns were simulated using cellular automaton: septal, RV free wall, LV pacing, and apical pacing and the extracellular potentials were calculated from the bidomain equation. An ellipsoidal cap was placed on a subsampled mesh of the epicardium to generate an enclosed pericardial mesh for the ECG forward simulation.

The cage dataset was generated from a torso tank experiment using a cardiac cage, which completely surrounds the heart with recording electrodes, therefore providing complete sampling of the sources, albeit farther from the heart. The recorded potentials on the cage were used as the sources of the forward simulation.

\subsection{Sampling strategies}

This study tested four sampling strategies of the atrial region of the heart (Figure 1). Sampling locations were first added in an increasing number to the atria staring near the AV plane (closest to the ventricular sock) and moving toward the atrial roof, in an inverted manner (from the atrial roof to the AV plane), in a combination of two locations, i.e., from the AV plane and atrial roof, and finally in randomly distributed order. The locations were added in 6 iterations for the KIT dataset, 7 for the CARP dataset, and 7 for the cage dataset.

\subsection{ECG Forward Simulation}

The ground truth data was calculated using an FEM forward simulation and sampled at the pericardial sock and torso surface. The ground truth pericardial or cage potentials were then sampled at the locations designated for each of the sampling strategies. The missing sampling locations were interpolated with laplacian interpolation to fill in the remaining cardiac surface potentials. BSPMs were simulated from the interpolated potentials using BEM and compared to the ground truth.

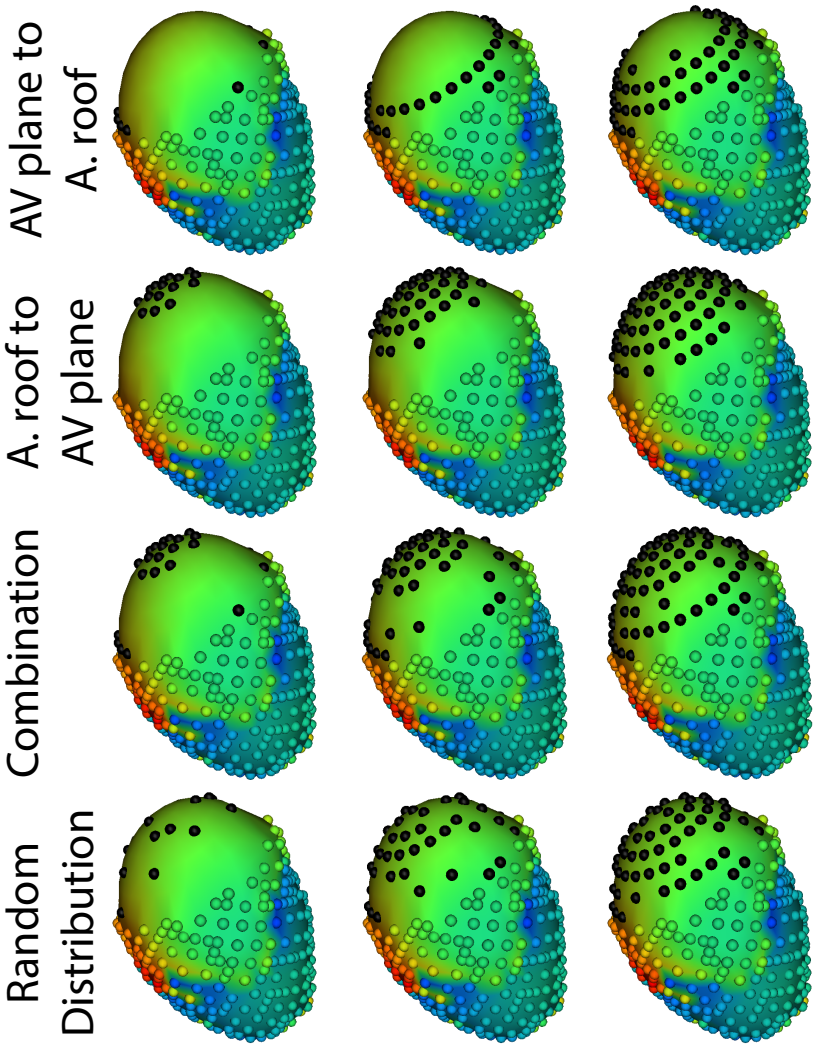

Figure 1. Cardiac source sampling strategies tested. Recording locations were added from the AV plane of the heart to the atrial roof, from the roof to the AV plane, a combination of the first two, and a random sampling of the atrial. Black marks indicate added atrial sampling locations.

\section{Results}

Using different sampling strategies for the atrial region of the heart altered the accuracy of the forward simulation depending on the number of added electrodes and the strategy used.

The absolute error of the forward simulation is reduced dramatically when using a full sampling of the epicardial surface compared to using source nodes covering only the ventricles with a mean reduction in the peak RMS error of $464 \mu \mathrm{V}$. Also as shown in Figure 2, there is global reduction in the magnitude of the absolute error and and a notable reduction of error in the anterior region of the body surface, resulting in more homogeneous distribution of error on the torso. Of note is the remaining error of the KIT dataset with full sampling of the cardiac surface, located near the apex of the heart.

A graded increase in the sampling of the atrial region yielded an asymptotic reduction in the peak error (Figure 3 ). The four sampling strategies performed similarly, 

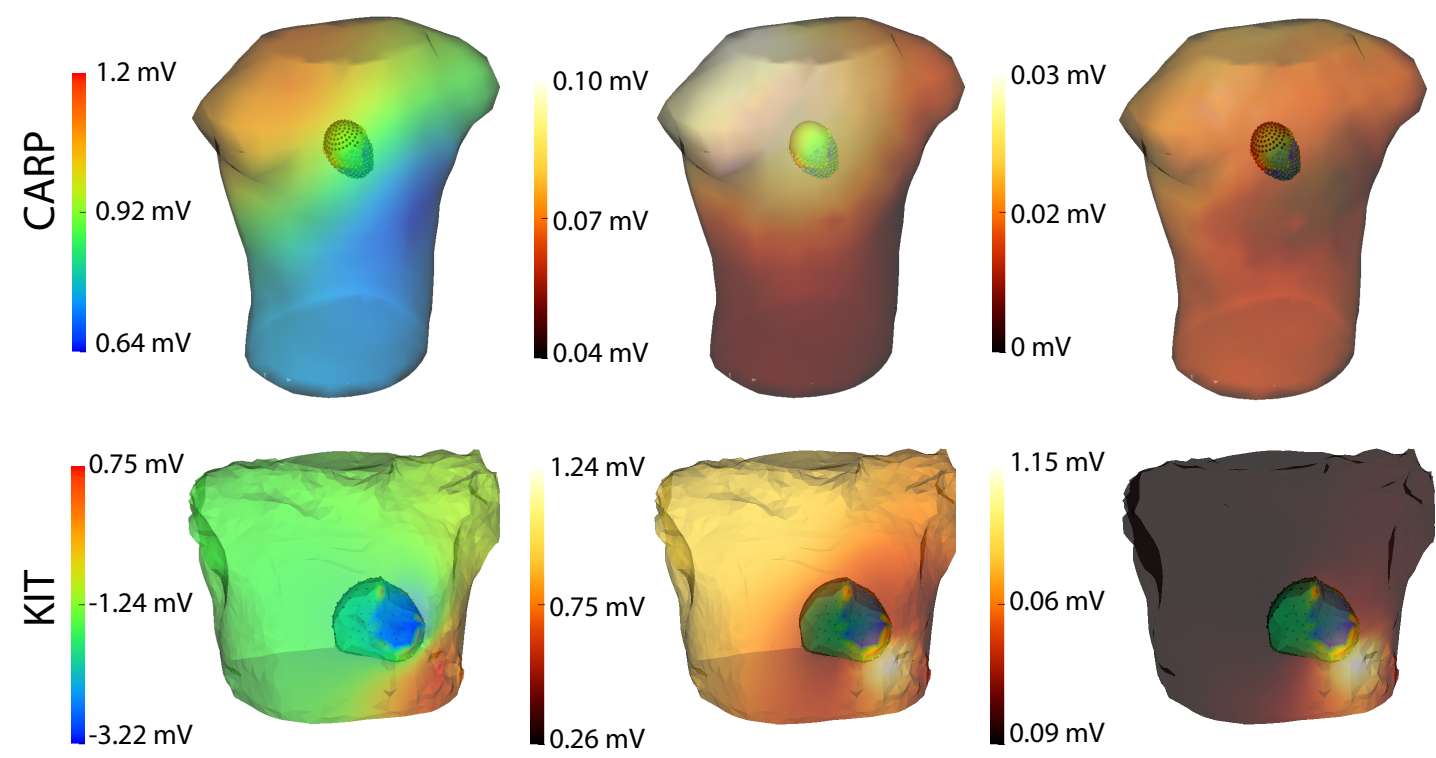

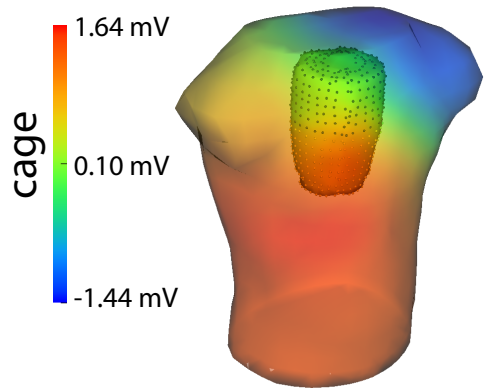

Ground Truth

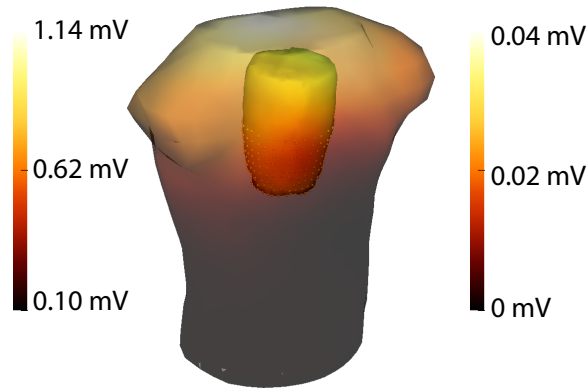

Ventricle Sampling Only

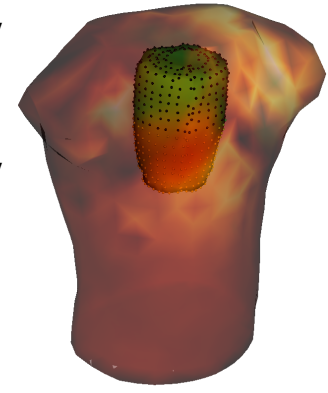

Full Sampling

Figure 2. Effect of atrial region sampling on forward simulation error. Shown is the ground truth potential map and the absolute error map of the forward simulation with sampling of the ventricles only and with full coverage of the ventricles and atria.

yet the randomly distributed and combined sampling approach had the most consistently lower peak RMS error. Of particular importance is the accuracy efficiency of the strategy, i.e., improving accuracy with fewer sampling locations, with which randomly distributed and combined sampling performed better than the other approaches.

\section{Discussion}

The results of this study indicate that sampling of the atrial region of the epicardium significantly impacts the forward simulation error. Omitting sampling on the atrial region can cause an error of similar magnitude to that found in validation studies that use epicardial socks [2], so it is possible that including sampling of the atrial region would reduce the error observed in these studies.

The results of this study also provide some insight into generating a strategy of placing recording electrodes in validation studies to mitigate errors that may arise from reduced sampling the atrial region of the heart. The random distribution showed the lowest error in most case; however distributed placements may be difficult to replicate in validation studies due to the many vessels near the atria. The combined approach also performed well in forward simulation accuracy, and this strategy would likely be easier to replicate in experimental studies. This strategy could be achieved by including a cluster of recording electrodes near the atrial roof with a plaque electrode and more sampling near the $\mathrm{AV}$ plane by increasing the size and coverage of the ventricular sock.

\section{Conclusions}

Since there are substantial errors in the ECG forward simulation resulting from using only ventricular epicardial potentials, future validation studies should incorporate ad- 

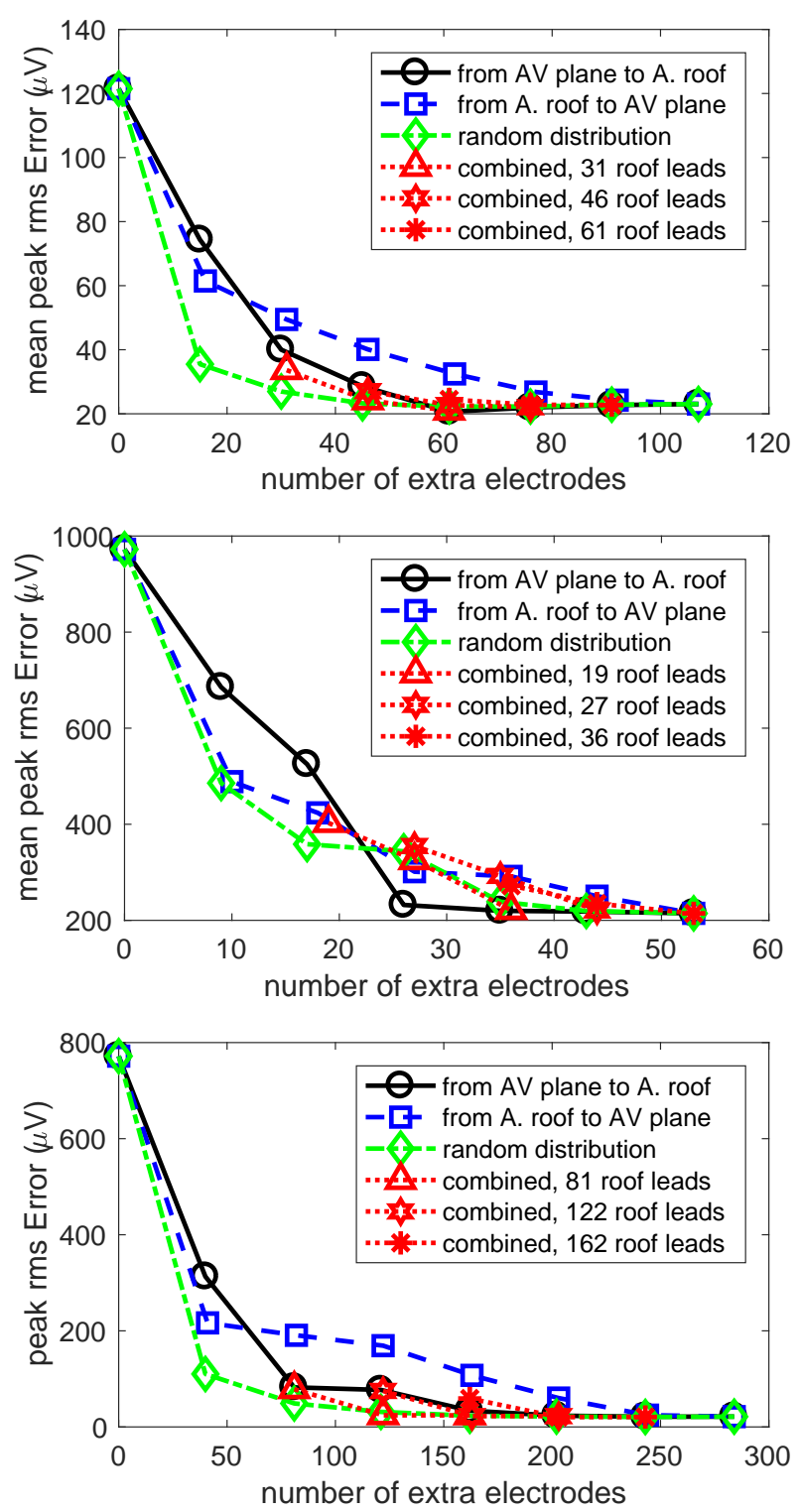

Figure 3. Peak RMS error of the forward simulation using different sampling strategies with increasing number of electrodes. From top to bottom the plots are the CARP, KIT, and cage dataset.

dition sampling of the atria by adding recording electrodes in that region. Any additional recordings in the region will reduce disagreement between simulated and recorded potentials. One practical and promising approach is to add electrodes to both the $\mathrm{AV}$ plane and atrial roof regions. Improving validation studies will show greater utility of the
ECG forward simulation and will also bolster the accuracy of and confidence in ECGI, which will improve noninvasive diagnoses of cardiac arrhythmias.

\section{Acknowledgements}

The research presented in this paper was made possible with help from the Cardiovascular Research and Training Institute (CVRTI) and the Nora Eccles Treadwell Foundation. This project was also supported by the National Institute of General Medical Sciences of the National Institutes of Health under grant number P41GM103545. Data used in this study was provided from a joint research project between the First Department of Medicine (Cardiology), University Medical Centre Mannheim and the Karlsruhe Institute of Technology (KIT).

\section{References}

[1] Barr R, Ramsey M, Spach M. Relating epicardial to body surface potential distributions by means of transfer coefficients based on geometry measurements. IEEE Trans Biomed Eng 1977;24:1-11.

[2] Bear LR, Cheng LK, LeGrice IJ, Sands GB, Lever NA, Paterson DJ, Smaill BH. The forward problem of electrocardiography: Is it solved? Circ Arrhythm Electrophysiol 2015;

[3] Shome S, MacLeod R. Simultaneous high-resolution electrical imaging of endocardial, epicardial and torso-tank surfaces under varying cardiac metabolic load and coronary flow. In Functional Imaging and Modeling of the Heart, Lecture Notes in Computer Science 4466. Springer-Verlag, 2007; 320-329.

[4] Vigmond E, Hughes M, Plank G, Leon L. Computational tools for modeling electrical activity in cardiac tissue. J Electrocardiol 2003;36 Suppl:69-74.

[5] Schulze WHW, Potyagaylo D, Schimpf R, Papavassiliu T, Tülümen E, Rudic B, Liebe V, Doesch C, Trächtler J, Borggrefe M, Dössel O. A simulation dataset for ecg imaging of paced beats with models for transmural, endoand epicardial and pericardial source imaging, 03 2015. URL http://doi.org/10.13140/RG.2.1.1946.8568.

Address for correspondence:

Jess Tate

Scientific Computing and Imaging Institute

WEB, 72 South Campus Drive, Rm 3750

Salt Lake City, UT 84112

jess@sci.utah.edu 